\title{
COVID-19: Clinical Presentation and Prognostic Factors of Severe Disease and Mortality in the Oldest-Old Population: A Cohort Study
}

\author{
César Gálvez-Barrón ${ }^{a, b}$ Marta Arroyo-Huidobro ${ }^{b}$ Antonio Miňarro ${ }^{c}$ \\ Gemma Añaños ${ }^{a}$ b Antonio Chamero ${ }^{a}$ Mireia Martín ${ }^{a}$ Clara Gris ${ }^{a}$ \\ Jose L. Avalos ${ }^{a}$ Anna M. Capielo ${ }^{a}$ Ester Ventosa ${ }^{a}$ Gemma Tremosa ${ }^{a}$ \\ Alejandro Rodríguez-Molinero ${ }^{\text {a, b }}$ COVID-19 research group of CSAPG \\ a'Research Area, Consorci Sanitari de l'Alt Penedès i Garraf (CSAPG), Sant Pere de Ribes, Barcelona, Spain; \\ ${ }^{b}$ Department of Geriatrics, Consorci Sanitari de l'Alt Penedès i Garraf (CSAPG), Sant Pere de Ribes, Barcelona, Spain; \\ 'Department of Genetics, Microbiology and Statistics, School of Biology, University of Barcelona, Barcelona, Spain
}

\section{Keywords}

COVID-19 · Oldest-old population · Mortality · Risk factors ·

Epidemiology

\begin{abstract}
Introduction: The oldest-old population (80 years or older) has the highest lethality from COVID-19. There is little information on the clinical presentation and specific prognostic factors for this group. This trial evaluated the clinical presentation and prognostic factors of severe disease and mortality in the oldest-old population. Methods: This is an ambispective cohort study of oldest-old patients hospitalized for respiratory infection associated with COVID-19 and with a positive test by RT-PCR. The clinical presentation and the factors associated with severe disease and mortality were evaluated (logistic regression). All patients were followed up until discharge or death. Results: A total of 103 patients (59.2\% female) were included. The most frequent symptoms were fever (68.9\%), dyspnoea (60.2\%), and cough (39.8\%), and $11.7 \%$ presented confusion. Fifty-nine patients (57.3\%) presented severe disease, and 59 died, with 43 patients (41.7\%) presenting both of these. In the multivariate analysis, female sex (odds ratio [OR] 0.31, 95\% confidence interval [95\% Cl]
\end{abstract}

$0.13-0.73, p 0.0074)$ and serum lactate dehydrogenase (LDH) (OR 2.55, 95\% Cl 1.21-5.37, $p$ 0.0139) were associated with severe disease, and serum sodium was associated with mortality (OR 3.12, 95\% Cl 1.18-8.26, p 0.0222). No chronic disease or pharmacological treatment was associated with worse outcomes. Conclusions: The typical presenting symptoms of respiratory infection in COVID-19 are less frequent in the oldest-old population. Male sex and LDH level are associated with severe disease, and the serum sodium level is associated with mortality in this population.

ㄷ) 2021 S. Karger AG, Basel

\section{Introduction}

The pandemic caused by the new severe acute respiratory distress syndrome coronavirus (SARS-CoV-2) represents a priority objective of current medical research, given its global extent. The older population has the highest lethality, having reported a crude fatality ratio of $12 \%$, and this is much higher (approximately $30 \%$ ) in the oldest-old people (80 or more years old) [1,2]. Given this population's greater vulnerability, knowledge of this pathology in them is a priority. karger@karger.com

www.karger.com/ger

Karger
(C) 2021 S. Karger AG, Basel

(2021 S. Karger AG, Basel
Correspondence to:

César Gálvez-Barrón, cgalvez@ csg.cat 
Proper management of affected oldest-old people requires knowledge of the clinical presentation and prognostic factors specific to this group. Most diseases, including infectious diseases, usually include atypical presentations, especially in this population [3-5], and their clinical profile means that the prognostic factors identified in the general adult population cannot be extrapolated.

Data on clinical presentation and prognostic factors have been reported in cohorts of older population [6]. However, most of these are from Asian populations and have a fairly low age cut-off (60-65 years), leaving the oldest-old population under-represented. The results of these studies cannot be extrapolated to the oldest-old population of our environment [7] since the level of autonomy and physical activity of the 60-70-year-old group is more similar to that of the youngest than the oldest patients $[7,8]$.

There are few studies specifically reporting on clinical presentation and prognostic factors in the oldest-old population [9-11]. The main reported symptoms of clinical presentation have included fever, dyspnoea, cough, and deterioration of functional status, and factors associated with higher mortality included age, male sex, severe functional dependence, cognitive decline, renal function, and inflammatory markers. However, other relevant variables like previous pharmacologic treatments or other important outcomes such as severe disease have not been specifically evaluated in this population. In the present study, we analysed the clinical presentation and the most important prognostic factors of severe disease and mortality in a cohort of oldest-old people (aged 80 years or more) hospitalized for COVID-19.

\section{Materials and Methods}

\section{Design and Sample}

The present work is a cohort study based on the previously described ambispective cohort $(n=464)$ of patients hospitalized for COVID-19 in the hospitals of the Consorci Sanitari de l'Alt Penedès i Garraf (CSAPG) [12]. The CSAPG includes 3 secondlevel hospitals with a total of 457 hospital beds, including 7 intensive care beds (extended to 24 beds at the peak of the epidemic) and 182 intermediate care beds. Its territorial scope includes an area of Barcelona with a reference population of 247,357 inhabitants.

For this study, patients aged 80 years or older who were admitted for respiratory infection associated with COVID-19 and with pharyngeal, nasal, or sputum smears positive for SARS-CoV-2 (RT-PCR) were included. All patients who were hospitalized through the emergency department were recruited from March 12 to May 2, 2020 and were followed up until hospital discharge or death. Patients with a positive COVID-19 test but without clinical or radiological respiratory involvement and patients with compatible respiratory symptoms who were treated as COVID-19 patients during admission but with negative smears ("COVID-19 clinical”) were excluded. Also excluded were patients who, despite meeting the diagnostic inclusion criteria, were not admitted to a hospitalization unit (e.g., due to death in the emergency department or transfer to a tertiary referral centre). In our case, there was no need to transfer patients to other centres of the same level for lack of hospital beds.

Patients were selected from the daily hospitalization census. This census included the medical diagnosis of admission of each patient and a signal that identified the patients who had requested an RT-PCR test for SARS-CoV-2. A predetermined calculation of the sample size was not performed. We included all possible patients who met the admission criteria.

\section{Variables and Information Collection}

Information on the variables was collected from the computerized medical records (GoWin program, version 2.4.0). The interviewers (the COVID-19 research group of the CSAPG [30 people]) began the study on April 6 and continued until the discharge or death of the last patient recruited. The information was collected with the help of 2 data collection notebooks (the first for baseline assessment and the second for the follow-up) created with the OpenClinica programme, version 3.14 (Copyright (C) OpenClinica LLC and collaborators, Waltham, MA, USA). Training sessions for data collection were held by the coordinating researcher of the study, and the quality control process included the review of at least $20 \%$ of the data of the main variables of the study to verify their agreement with the source document. If necessary, retraining and supervision sessions were held.

In the baseline assessment, sociodemographic, comorbidity, previous pharmacological treatment, and clinical presentation data were collected from the emergency assessment data. The data of comorbidity and previous pharmacological treatments were collected after reviewing all the medical reports available in the computerized clinical history. We recorded the data categorically (yes/no) from a predetermined list prepared by the researchers (Table 1).

The clinical presentation variables were collected from the emergency department medical report and included symptoms and signs (categorically recorded from a predetermined list), oxygen saturation, pulmonary radiological involvement (number of affected lung quadrants, range $0-4$ ), and the level of C-reactive protein (CRP) (hereinafter "emergency CRP"). During each day of the follow-up, the following variables were collected: hospital discharge, oxygenation system (nasal cannulas, mask, non-rebreather mask, non-invasive mechanical ventilation, and orotracheal intubation), and death. For the present study, the laboratory parameters of the first day of hospitalization were also considered, which were extracted automatically by the Department of Informatics to avoid manual registration errors.

The variables considered potentially prognostic were those collected in the baseline assessment and the laboratory parameters of the first day of hospitalization. The outcome variables were 2: mortality and severe disease, which were verified every day of the follow-up. The standard definition of severe disease (dyspnoea, a respiratory rate of 30 or more breaths per minute, a blood oxygen saturation of $93 \%$ or less, a ratio of the partial pressure of arterial 
Table 1. Baseline assessment of the patients included in the study

\begin{tabular}{|c|c|c|}
\hline Variable & Total & $N(\%)$ \\
\hline \multicolumn{3}{|l|}{ Block 1: personal background } \\
\hline \multicolumn{3}{|l|}{ Sociodemographic } \\
\hline Female sex & 103 & $61(59.2)$ \\
\hline Age, years (SD) & 103 & $86.75(4.65)$ \\
\hline Institutionalized & 103 & $63(61.2)$ \\
\hline \multicolumn{3}{|l|}{ Autoimmune } \\
\hline Rheumatoid arthritis & 103 & 0 \\
\hline SLE & 103 & 0 \\
\hline Spondyloarthropathies & 103 & 0 \\
\hline Scleroderma & 103 & 0 \\
\hline Psoriasis & 103 & 0 \\
\hline Other autoimmune disease & 103 & $7(6.8)$ \\
\hline \multicolumn{3}{|l|}{ Renal } \\
\hline Chronic kidney failure & 103 & $34(33.0)$ \\
\hline Peritoneal dialysis & 103 & 0 \\
\hline Haemodialysis & 103 & $1(1.0)$ \\
\hline \multicolumn{3}{|l|}{ Cardiovascular disease or risk factors } \\
\hline Hypertension & 103 & $84(81.6)$ \\
\hline Diabetes mellitus 2 & 103 & $35(34.0)$ \\
\hline Dyslipidaemia & 103 & $42(40.8)$ \\
\hline Obesity & 103 & $13(12.6)$ \\
\hline Smoking & 103 & $5(4.9)$ \\
\hline Alcoholism & 103 & $1(1.0)$ \\
\hline Heart failure & 103 & $17(16.5)$ \\
\hline Atrial fibrillation & 103 & $24(23.3)$ \\
\hline Ischaemic heart disease & 103 & $16(15.5)$ \\
\hline Other arterial ischaemia & 103 & $2(1.9)$ \\
\hline Aortic valve disease & 103 & $8(7.8)$ \\
\hline Mitral valve disease & 103 & $7(6.8)$ \\
\hline Prosthetic cardiac valve & 103 & 0 \\
\hline Other heart disease & 103 & $6(5.8)$ \\
\hline Pacemaker carrier & 103 & $4(3.9)$ \\
\hline Stroke & 103 & $13(12.6)$ \\
\hline Pulmonary hypertension & 103 & $2(1.9)$ \\
\hline \multicolumn{3}{|l|}{ Psychiatric } \\
\hline Depression & 103 & $25(24.3)$ \\
\hline Schizophrenia & 103 & $1(1.0)$ \\
\hline Other psychiatric diseases & 103 & $10(9.7)$ \\
\hline \multicolumn{3}{|l|}{ Neurodegenerative diseases } \\
\hline Dementia & 103 & $36(35.0)$ \\
\hline Parkinson disease & 103 & $2(1.9)$ \\
\hline Multiple sclerosis & 103 & 0 \\
\hline Other neurodegenerative diseases & 103 & $4(3.9)$ \\
\hline \multicolumn{3}{|l|}{ Digestive } \\
\hline Gastropathy & 103 & $7(6.8)$ \\
\hline Inflammatory bowel disease & 103 & $4(3.9)$ \\
\hline Cirrhosis & 103 & 0 \\
\hline Celiac disease & 103 & 0 \\
\hline Other liver disease & 103 & $4(3.9)$ \\
\hline \multicolumn{3}{|l|}{ Respiratory } \\
\hline Asthma & 103 & $3(2.9)$ \\
\hline COPD & 103 & $16(15.5)$ \\
\hline Cystic fibrosis & 103 & 0 \\
\hline Other pneumopathy & 103 & $4(3.9)$ \\
\hline
\end{tabular}


Table 1 (continued)

\begin{tabular}{|c|c|c|}
\hline Variable & Total & $N(\%)$ \\
\hline \multicolumn{3}{|l|}{ Other } \\
\hline Thyroid disease & 103 & $13(12.6)$ \\
\hline HIV/AIDS & 103 & 0 \\
\hline Organ transplant & 103 & 0 \\
\hline Immunosuppression due to other causes & 103 & 0 \\
\hline Chronic anaemia & 103 & $12(11.7)$ \\
\hline $\mathrm{HCV}$ & 103 & 0 \\
\hline \multicolumn{3}{|l|}{ Block 2: pharmacological treatments } \\
\hline \multicolumn{3}{|l|}{ Haematological } \\
\hline Antiplatelet agents & 103 & $33(32.0)$ \\
\hline Anticoagulants & 103 & $17(16.5)$ \\
\hline \multicolumn{3}{|l|}{ Analgesics and corticosteroids } \\
\hline Paracetamol & 103 & $43(41.7)$ \\
\hline NSAIDs & 103 & $8(7.8)$ \\
\hline Opioids & 103 & $13(12.6)$ \\
\hline Systemic corticosteroids & 103 & $5(4.9)$ \\
\hline \multicolumn{3}{|l|}{ Antidiabetic } \\
\hline Insulin & 103 & $10(9.7)$ \\
\hline Metformin & 103 & $20(19.4)$ \\
\hline Other oral antidiabetic drugs & 103 & $9(8.7)$ \\
\hline \multicolumn{3}{|l|}{ Cardiovascular } \\
\hline Lipid-lowering drugs & 103 & $26(25.2)$ \\
\hline Diuretics & 103 & $46(44.7)$ \\
\hline Beta blockers & 103 & $17(16.5)$ \\
\hline ACE inhibitors & 103 & $30(29.1)$ \\
\hline ARA 2 & 103 & $21(20.4)$ \\
\hline Other antihypertensives & 103 & $31(30.1)$ \\
\hline Antiarrhythmics & 103 & $9(8.7)$ \\
\hline \multicolumn{3}{|l|}{ Respiratory } \\
\hline Inhaled anticholinergics & 103 & $12(11.7)$ \\
\hline$\beta_{2}$ inhaled agonists & 103 & $14(13.6)$ \\
\hline Inhaled corticosteroids & 103 & $10(9.7)$ \\
\hline Other inhalers & 103 & $2(1.9)$ \\
\hline Home oxygen therapy & 103 & $4(3.9)$ \\
\hline \multicolumn{3}{|l|}{ CNS } \\
\hline Sedatives & 103 & $32(31.1)$ \\
\hline Antidepressants & 103 & $41(39.8)$ \\
\hline Antipsychotics & 103 & $30(29.1)$ \\
\hline Anti-epileptics & 103 & $4(3.9)$ \\
\hline Antiparkinsonians & 103 & $4(3.9)$ \\
\hline Other drugs with effect on CNS & 103 & $14(13.6)$ \\
\hline \multicolumn{3}{|l|}{ Other therapies } \\
\hline Antacids & 103 & $51(49.5)$ \\
\hline Cytotoxic/chemotherapy & 103 & 0 \\
\hline Drugs with immune action & 103 & 0 \\
\hline Antihistamines & 103 & $1(1.0)$ \\
\hline
\end{tabular}


Table 1 (continued)

\begin{tabular}{|c|c|c|}
\hline Variable & Total & $N(\%)$ \\
\hline \multicolumn{3}{|l|}{ Block 3: clinical presentation } \\
\hline Fever & 103 & $71(68.9)$ \\
\hline Dyspnoea & 103 & $62(60.2)$ \\
\hline Cough & 103 & $41(39.8)$ \\
\hline Diarrhoea & 103 & $16(15.5)$ \\
\hline Arthromyalgia & 103 & $6(5.8)$ \\
\hline Asthenia & 103 & $22(21.4)$ \\
\hline Anosmia & 103 & 0 \\
\hline Altered taste & 103 & 0 \\
\hline Skin lesions & 103 & 0 \\
\hline Headache & 103 & 0 \\
\hline Confusion & 103 & $12(11.7)$ \\
\hline Psychomotor agitation, $\%$ & 103 & $3(2.9)$ \\
\hline Chest X-ray (affected quadrants) & 103 & \\
\hline 0 & & $12(12.6)$ \\
\hline 1 & & $15(15.8)$ \\
\hline 2 & & $36(37.9)$ \\
\hline 3 & & $15(15.8)$ \\
\hline 4 & & $17(17.9)$ \\
\hline CRP, mg/L in emergencies, mean (SD) & 34 & $151.03(110.72)$ \\
\hline Basal oxygen saturation (emergency), mean (SD) & 93 & $86.82(10.56)$ \\
\hline Block 4: laboratory parameters (day 1 of admission) & $\mathrm{n}$ & Mean (SD) \\
\hline Haemoglobin, g/dL & 87 & $12.53(2.21)$ \\
\hline Platelets, $10^{9} / \mathrm{L}$ & 85 & $232.79(117.17)$ \\
\hline Neutrophils, $10^{9} \mathrm{~L}$ & 82 & $7.27(4.38)$ \\
\hline Lymphocytes, $10^{9} / \mathrm{L}$ & 87 & $1.16(0.89)$ \\
\hline Eosinophils, $10^{9} / \mathrm{L}$ & 87 & $0.32(0.63)$ \\
\hline Prothrombin time (INR) & 84 & $1.28(0.48)$ \\
\hline $\mathrm{D}$-dimer, $\mathrm{ng} / \mathrm{mL}$ & 71 & $2,842.82(3,468.59)$ \\
\hline Fibrinogen, $\mathrm{mg} / \mathrm{dL}$ & 15 & $614.67(242.51)$ \\
\hline Glucose, $\mathrm{mg} / \mathrm{dL}$ & 87 & $149.44(64.36)$ \\
\hline Sodium, $\mathrm{mEq} / \mathrm{L}$ & 87 & $141.64(8.25)$ \\
\hline $\mathrm{Cr}, \mathrm{mg} / \mathrm{dL}$ & 87 & $1.49(0.92)$ \\
\hline Urea, mg/dL & 87 & $78.36(54.11)$ \\
\hline Alkaline phosphatase, IU/L & 50 & $73.99(30.42)$ \\
\hline AST, IU/L & 60 & $44.85(44.22)$ \\
\hline ALT, IU/L & 66 & $28.15(14.57)$ \\
\hline GGTP, IU/L & 53 & $50.57(27.47)$ \\
\hline Total bilirubin, $\mathrm{mg} / \mathrm{dL}$ & 70 & $0.66(0.49)$ \\
\hline $\mathrm{LDH}, \mathrm{U} / \mathrm{L}$ & 73 & $325.01(131.48)$ \\
\hline $\mathrm{CRP}$ at admission, $\mathrm{mg} / \mathrm{L}$ & 80 & $13.37(10.83)$ \\
\hline Ferritin, $\mu \mathrm{g} / \mathrm{L}$ & 48 & $518.44(491.55)$ \\
\hline Procalcitonin, $\mathrm{ng} / \mathrm{mL}$ & 30 & $0.80(1.34)$ \\
\hline Lactate, $\mathrm{mmol} / \mathrm{L}$ & 20 & $2.14(1.79)$ \\
\hline Arterial oxygen, $\mathrm{mm} \mathrm{Hg}$ & 61 & $73.61(30.30)$ \\
\hline Carbon dioxide, $\mathrm{mm} \mathrm{Hg}$ & 61 & $24.45(4.32)$ \\
\hline Serum bicarbonate, $\mathrm{mmol} / \mathrm{L}$ & 71 & $24.79(3.38)$ \\
\hline $\mathrm{pH}$ & 61 & $7.46(0.05)$ \\
\hline
\end{tabular}

SD, standard deviation; SLE, systemic lupus erythematosus; COPD, chronic obstructive pulmonary disease; $\mathrm{HIV}$, human immunodeficiency virus; $\mathrm{HCV}$, hepatitis $\mathrm{C}$ virus; NSAIDs, non-steroidal anti-inflammatory drugs; ACE inhibitors, inhibitors of the angiotensin-converting enzyme; ARA 2, angiotensin 2 receptor antagonists; CNS, central nervous system; CRP, C-reactive protein; INR, international normalized ratio; AST, aspartate aminotransferase; ALT, alanine aminotransferase; GGTP, gamma-glutamyl transpeptidase; LDH, lactate dehydrogenase. 
oxygen to the fraction of inspired oxygen of $<300 \mathrm{~mm} \mathrm{Hg}$, or infiltrates in $>50 \%$ of the lung field) [13] was considered too broad for our study since the majority of hospitalized patients would meet the criteria of this definition. For this reason, in this study, severe disease was defined as the need for oxygen therapy with a reservoir mask, mechanical ventilation (invasive or non-invasive), or high-flow nasal cannulas. This definition is similar to the categories 6-9 of the Clinical Progression Scale of the World Health Organisation [14].

Regarding the prognostic factor-outcome variable association, age and sex were considered a priori as potential confounding variables and/or effect modifiers of all other variables evaluated. The data collection notebooks with the complete lists of variables are available in see online suppl. material 1 and 2; for all online suppl. material, see www.karger.com/doi/10.1159/000515159.

\section{Statistical Analysis}

For the analysis of the prognostic factors of death and severe disease, the potential prognostic variables were grouped into 4 blocks: age-sex-comorbidity (block 1); previous pharmacological treatment (block 2); variables of clinical presentation, including pulmonary radiological involvement and CRP in the emergency room (block 3); and variables of laboratory parameters (block 4). Within each block and for each outcome variable, a bivariate analysis was performed with each prognostic variable ( $\chi^{2}$ or Fisher's test for categorical variables, the $T$-test or Mann-Whitney test for quantitative variables), and a multivariate model was built using logistic regression, except for block 3 (in this block, it was considered more relevant to evaluate the individual prognostic capacity of each parameter).

In the bivariate analysis and given the multiplicity of analyses performed, the statistical significance was adjusted by the false discovery rate method [15]. In all the planned multivariate models, age and sex were included, given their status as potential confounding variables. The variables with significant associations (unadjusted $p<$ 0.05 ) found in the bivariate analysis were preselected for the models. As the primary objective of our study was the identification of the prognostic factors with high associative strength and considering the high number of potential prognostic factors to be evaluated, the least absolute shrinkage and selection operator (LASSO) method was used for the final selection of the variables to be included in the models. The LASSO method [16] is not based on $p$ values (which could induce the inclusion of superfluous clinical variables in the final model) but on a modification of the minimum quadratic estimation. Its objective is to select a smaller subset of explanatory variables (but with greater strength of association) with which to finally adjust the model without significantly losing any explanatory quality of the model. This procedure is considered superior to eliminating the prognostic variables according to $p$ value, and it reduces the risk of multicollinearity problems, which may arise in models with a large number of potential prognostic variables [17]. Variables with $>30 \%$ missing values were excluded from the multivariate models, as were those with 15 or fewer individuals with the evaluated condition. Finally, based on the results of the bivariate analysis and to avoid collinearity, $\mathrm{Cr}$ was excluded from the models when it coincided in the preselection with the urea variable.

Quantitative variables were not categorized. The laboratory parameters were transformed logarithmically to improve their fit to a normal distribution and were scaled to allow a comparison of their odds ratios (ORs).

COVID-19 in the Oldest-Old Population
Regarding the missing data, in case there were no laboratory parameters from the first day of hospitalization, these variables were imputed from their values of the second day of hospitalization if the latter were available. No missing data of other variables were imputed. R version 3.6.1 (R Project for Statistical Computing) and IBM SPSS version 26 were used.

\section{Results}

During the recruitment period, 464 people (113 aged 80 or older) were hospitalized for suspected infection with COVID-19, of whom 418 had respiratory infection with a positive RT-PCR test for SARS-CoV-2. Of this last group, 89 people $(21 \%)$ were aged 80 or more years. Additionally, 14 patients aged 80 years or older identified after a second review of the hospitalization censuses were included, so 103 oldest-old people were included in the end.

The baseline assessment data are shown in Table 1. The mean age was 86.75 (standard deviation [SD] 4.65; maximum age 99 ) years. Sixty-one patients (59.2\%) were female, and $63(61.2 \%)$ came from a nursing home (institutionalized), and 99 patients $(96.1 \%)$ had 2 or more chronic diseases. The most frequent symptoms of clinical presentation were fever $(68.9 \%)$, dyspnoea $(60.2 \%)$, and cough $(39.8 \%)$.

All patients were followed up until discharge or death. The median follow-up was 6.0 (interquartile range 8) days for the whole sample, 11 days for who survived, 5 days for who presented severe disease criteria, and finally 3 days for who died. Fifty-nine patients (57.3\%) had severe disease, and 59 patients died, with both events occurring in 43 patients. In 16 patients who died (1.1 out of every 4 patients who died), no criteria for severe disease were previously detected. In order to exclude medical indication for exclusive palliative care including palliative sedation as cause for not detecting criteria of severe disease, we reviewed individually these cases, and only 2 patients with medical indication for exclusive palliative care were detected.

The results of the bivariate analysis are shown in Table 2. (Table with more extensive data on bivariate analysis including the number of patients of each group is available as supplementary material.) Significant prognostic variables (unadjusted $p$ value) were found for both outcome variables only in the blocks of clinical presentation variables (dyspnoea, radiological involvement, and oxygen saturation) and of laboratory parameters (aspartate aminotransferase [AST], lactate dehydrogenase [LDH], and CRP on admission), although out of all of 


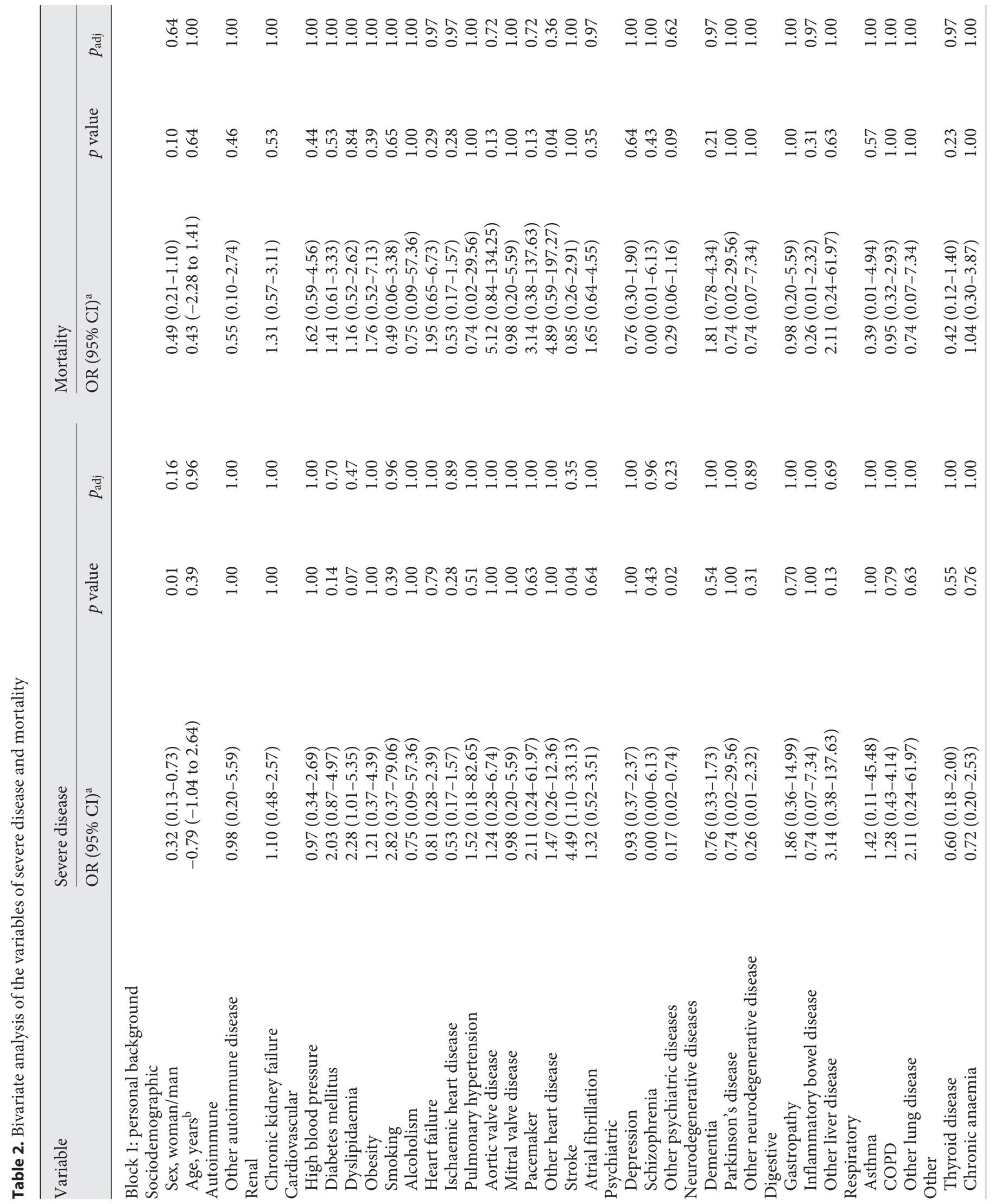




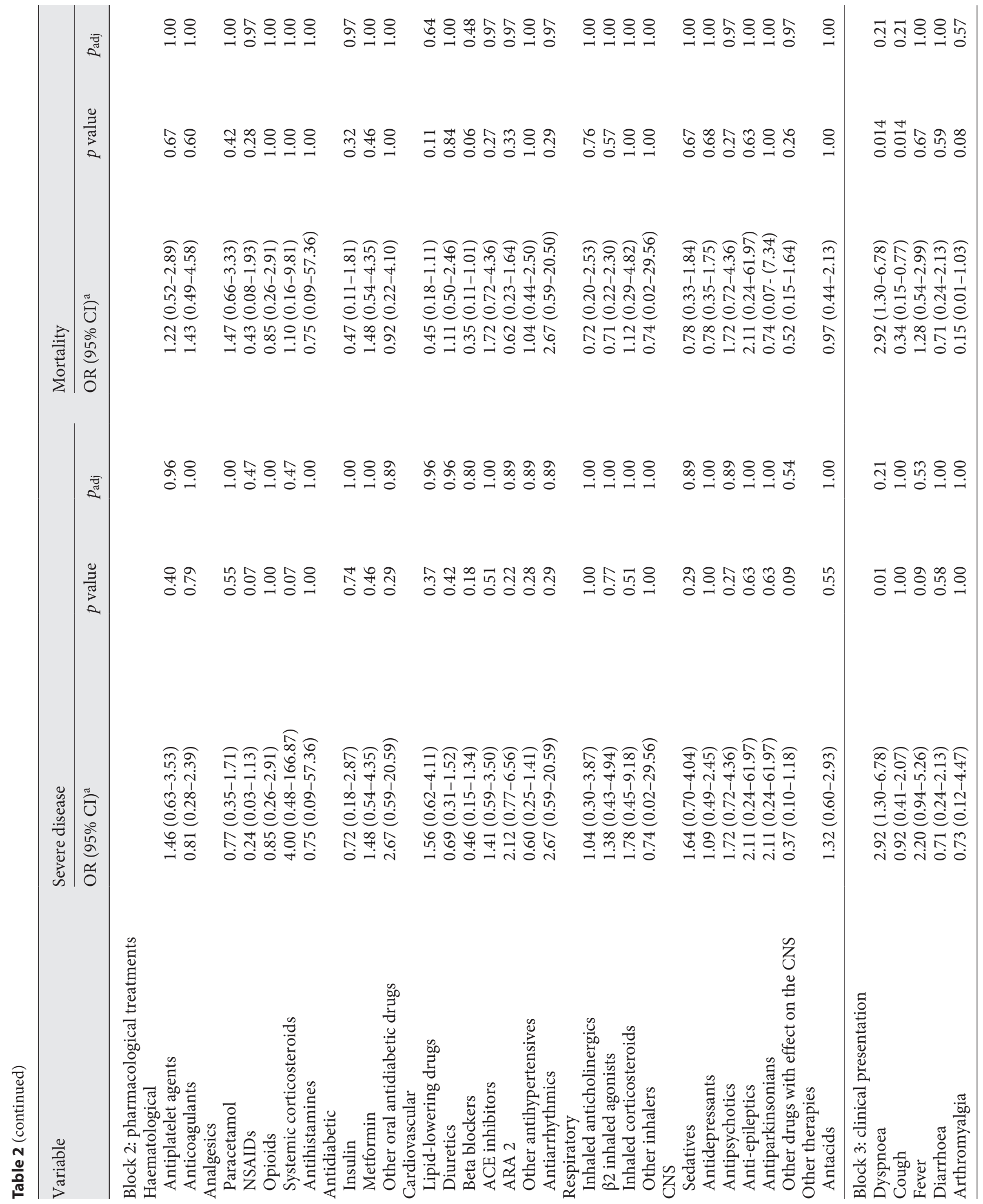




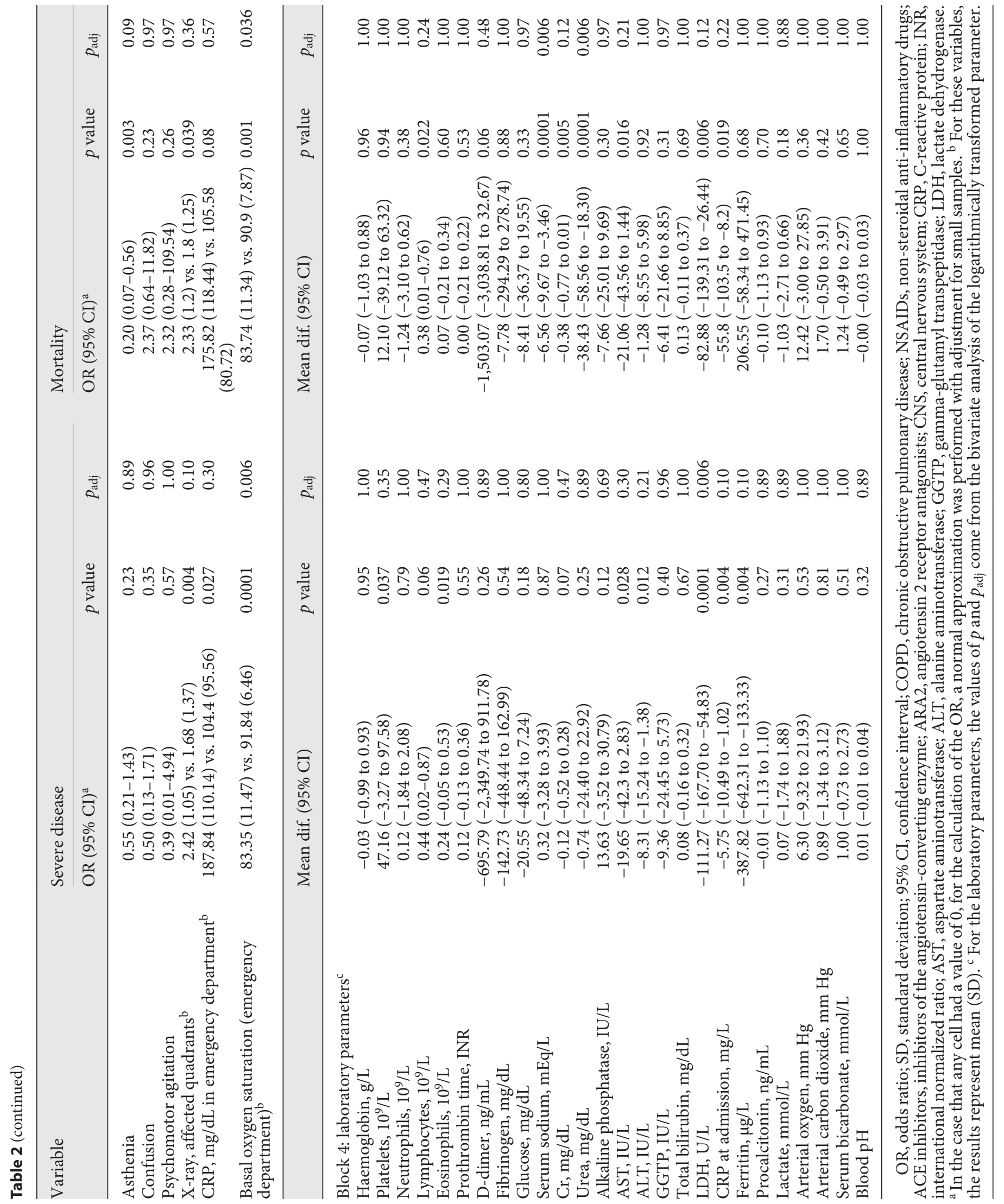


Table 3. Results of the multivariate analysis for the variables of severe disease and mortality

\begin{tabular}{|c|c|c|c|c|c|c|}
\hline Model $^{\mathrm{a}}$ & \multicolumn{3}{|c|}{ Severe disease } & \multicolumn{3}{|c|}{ Mortality } \\
\hline \multicolumn{7}{|l|}{ Model 1: Personal history } \\
\hline Female sex & 0.31 & $0.13-0.73$ & 0.007 & & & \\
\hline \multicolumn{7}{|c|}{ Model 2: Pharmacological treatments } \\
\hline Female sex & 0.31 & $0.13-0.73$ & 0.007 & 0.48 & $0.21-1.10$ & 0.082 \\
\hline Serum LDH & 2.55 & $1.21-5.37$ & 0.014 & 1.65 & $0.88-3.10$ & 0.121 \\
\hline CRP on admission & 1.93 & $0.89-4.17$ & 0.094 & & & \\
\hline Serum sodium & & & & 3.12 & $1.18-8.26$ & 0.022 \\
\hline Serum urea & & & & 1.49 & $0.66-3.39$ & 0.339 \\
\hline Lymphocytes & & & & 0.78 & $0.39-1.56$ & 0.484 \\
\hline \multicolumn{7}{|c|}{ Model 4: Laboratory parameters and oxygen saturation } \\
\hline Serum sodium & & & & 2.60 & $1.05-6.44$ & 0.0394 \\
\hline Serum urea & & & & 1.54 & $0.70-3.38$ & 0.281 \\
\hline
\end{tabular}

OR, odds ratio; 95\% CI, confidence interval; CRP, C-reactive protein; LDH, lactate dehydrogenase. ${ }^{\text {a }}$ In each model, the variables age and sex were included, except in model $4 .{ }^{\mathrm{b}}$ Laboratory parameters (LDH, CRP, sodium, urea, and lymphocytes) were subjected to logarithmic transformation and then scaled, so their ORs cannot be compared with those of the other variables (sex and oxygen saturation).

them, only oxygen saturation survived the adjustment of multiple tests (false discovery rate method). The urea and sodium parameters were significantly associated with mortality.

The results of the multivariate models are shown in Table 3. By the criterion of $>30 \%$ missing data or fewer than 16 positive individuals with the evaluated parameter, the variables of stroke, psychiatric disease, emergency CRP, AST, alanine aminotransferase, and ferritin were excluded from the severe disease models, and the variables AST and "other heart disease" were excluded from the mortality models. With respect to the outcome severe disease, the variable female sex (OR 0.31, 95\% confidence interval $[95 \% \mathrm{CI}] 0.13-0.73, p 0.0074)$ was significantly associated with it in block 1 , as was serum LDH (OR 2.55, 95\% CI 1.21-5.37, p 0.0139) in block 4 . Regarding the mortality outcome, only serum sodium (block 4) was significantly associated with it (OR 3.12, $95 \%$ CI 1.18-8.26, $p$ 0.0222). Given this last result, we built a model including laboratory parameters and oxygen saturation. In this model, serum sodium continued to be associated with higher mortality (OR 2.60, 95\% CI $1.05-6.44, p 0.0394)$.

COVID-19 in the Oldest-Old Population

\section{Discussion/Conclusion}

\section{Main Results}

The most frequent symptoms of clinical presentation were fever, dyspnoea, and cough; hospital mortality was quite high; male sex and serum LDH level were associated with severe disease; and serum sodium concentration was associated with mortality.

\section{Clinical Presentation}

Although the most frequent symptoms were the same as in the hospitalized adult population [12, 18-20], they were less frequent than has been reported in this population (especially cough and fever), even though the diagnosis of respiratory infection was an inclusion criterion in our study. In general population, cohorts of COVID19-hospitalized patients [18-20] have reported prevalence of fever and cough between 72 and 88 and between 65 and $73 \%$, respectively. On the other hand, confusion stands out as a symptom present in $11 \%$ of our sample. Gutiérrez-Rodríguez et al. [11] and Annweiler et al. [21] reported frequencies similar to ours in the subgroup of patients 80 years or older. These findings are in line with those observed in the majority of diseases of this popula- 
tion (including infections), in which less symptomatic or atypical presentations are more frequently observed [35]. This reinforces the need for a lower suspicion threshold in this population, especially when evaluated in an emergency department.

A low frequency of non-respiratory symptoms was observed. However, given the absence of a systematic search for these symptoms, we cannot exclude an information bias whereby patients who reported respiratory symptoms at the beginning of the evaluation were not also asked about non-respiratory symptoms, resulting in undetected symptoms.

\section{Prognostic Factors of Severe Disease and Mortality}

Age, unlike in the younger population $[2,6,22,23]$, did not bring an added risk in any of the models built here, and male sex was associated with severe disease but not mortality. The trials by Gutiérrez-Rodríguez et al. [11] and Covino et al. [10] did not find that age or sex was associated with mortality either. However, Ramos-Rincon et al. [9] reported age and male sex as variables associated with mortality from a multicentre cohort of 2,772 very old hospitalized patients, so we cannot rule out a lack of statistical power of our sample in these results. Institutionalization was not included in our analysis due to the fact that the registration of this variable was not as reliable as the other included variables. Furthermore, we consider that institutionalization, in contrast to variables like chronic diseases or previous pharmacological treatments, is an external indicator, instead of being an intrinsic factor of a patient. For this reason, the evaluation of institutionalization as a prognostic factor (in difference to its evaluation as predictor factor) might not be recommended.

Unlike laboratory parameters and those related to emergency assessment (some of which were associated with worse prognosis), no chronic disease or previous pharmacological treatment was associated with worse or better outcomes. Previous treatments have not been evaluated as prognostic factors in the aforementioned trials [9-11], so we cannot compare our results with them. In a younger cohort, Mostaza et al. [24], in people older than 75 years, did find a better prognosis in patients who previously took renin-angiotensin-aldosterone system antagonists. Regarding previous chronic diseases, it is remarkable that in contrast to studies in younger populations [25], no chronic disease was associated with mortality. Although we cannot exclude a lack of statistical power for this result, it is similar to the study by RamosRincon et al. [9], which also did not find an association between chronic diseases and mortality (except for obe- sity which was significantly associated with mortality in the bivariant analysis, although it was not included in the multivariant models). In another study with a very old population, Covino et al. [10] reported severe dementia as an independent risk factor for death, although age, since it was not apparently included in their multivariate analysis, cannot be rule out as a confounding factor.

Only the serum LDH level was associated with severe disease among laboratory parameters. We have not found studies that evaluated the factors associated with this result in the older or oldest-old population. In adult population studies, $\mathrm{LDH}$ is one of the most powerful factors associated with severe disease among laboratory parameters [26-29]. Thus, in the meta-analysis of Zhang et al. [28], LDH was the only laboratory parameter associated with both adult respiratory distress syndrome and indication for intensive care unit admission. $\mathrm{LDH}$ is present in body tissues and is released from damaged cells [30, 31], increases lactate production [32], and is a good predictor of lung injury [30].

Serum sodium was associated with mortality in our sample. Of the aforementioned studies, Gutiérrez-Rodríguez et al. [11] did not find a significant association in their bivariate analysis, and Ramos-Rincon et al. [9] and Covino et al. [10] did not include this parameter in their reports. Below, we propose a hypothesis for this result.

Among the laboratory parameters, it stands out that the parameters associated with worse prognosis were different for severe disease and mortality, although both outcome variables are closely related. We highlight the fact that 1.1 out of every 4 people who died did not previously present any criteria of severe disease. Given the definition of this in our study based on strict respiratory criteria, we hypothesize that some of the patients could have died from complications in other body systems (cardiovascular, thrombotic, metabolic, and renal complications have been described [33]) and not so much from severe respiratory involvement. In this sense, serum sodium (a marker of metabolic alteration or renal function) would predominate as a prognostic factor for mortality and not as much for severe respiratory disease. That the association of this parameter with mortality was maintained, despite adjusting the model for oxygen saturation reinforces our hypothesis.

With respect to our analyses of severe disease, we have to comment the possibility of competing risk factors when the patients died without severe disease criteria (in these patients, dead competes with the severe disease outcome). This would be significant if the proportion of patients who died without severe disease criteria had been similar or larger than the proportion of patients with se-
Gálvez-Barrón et al. 
vere disease, or if the follow-up time had been very long ( $>5$ years) [34]. In our study, the proportion of patients who died without severe disease criteria (15.5\%) was significantly lower than the patients with severe disease (57.3\%), and the follow-up time of our cohort was very short. Thus, we consider the impact of a situation of competing risk factors insignificant or improbable in our trial.

\section{In-Hospital Mortality}

Although the highest lethality of COVID-19 is seen in the older population, especially among the hospitalized population, the hospital mortality found in our sample was higher than that of other hospital series of oldest-old populations in Spain (35-47\%). We highlight the high proportion of institutionalized patients in our sample (61\%), reflecting a population with greater clinical fragility and therefore with less ability to respond to an organic stressor. Thus, the series reported by Gutiérrez-Rodríguez et al. [11] had a mortality (41\%) and a proportion of institutionalized patients (70\%) more similar to those of our sample than those reported by Mostaza et al. [24] (mortality 35\% and proportion of institutionalized 23\%). In neighbouring countries, Zerah et al. [35] (France) reported a lower lethality (31\%) in a cohort of 821 hospitalized patients aged 70 years or older, although with a proportion of institutionalized patients much lower than that in our sample (29\%). Finally, we have to mention, in relation to the high mortality in our sample, the high pressure to which the Spanish Sanitary System was exposed during this time, which might have influenced or limited the access for this population group to certain health care resources including admission to intensive care units [36].

\section{External Validity}

Regarding the extrapolation or comparison of our results with the results in other samples, it is important to consider, in addition to the high mortality and high proportion of institutionalized patients, that our patients were managed in secondary referral centres, so our results cannot be extrapolated to populations treated on an outpatient basis or in centres of maximum complexity (tertiary), such as patients undergoing organ transplants. Furthermore, in our sample, only 4 patients had less than 2 chronic diseases, which means that our results cannot be extrapolated to patients without significant comorbidity.

\section{Limitations}

The sample size of our study does not allow us to take the results as conclusive. Some laboratory parameters (AST, aspartate aminotransferase, ferritin, and $\mathrm{Cr}$ ), despite hav- ing significant associations with some of the outcome variables in the bivariate analysis, could not be included in the multivariate models due to significant data loss.

Most likely, some COVID-19 patients were already admitted with severe disease criteria. In these cases, the validity of our results regarding the variables of clinical presentation and laboratory parameters (including serum LDH level) may be affected. The sample size prevented us to perform any sensitivity analysis whereby we recognize this limitation. However, the variables of chronic diseases and previous treatments would continue to be valid in these patients because the temporal relationship remains accurate. Finally, we were unable to evaluate variables of previous functional status, a variable of known prognostic association in most diseases in this population, including COVID-19 [35, 37].

In conclusion, the symptoms of clinical presentation typical of respiratory infection by SARS-CoV-2 (fever, dyspnoea, and cough) are less frequent in the oldest-old population, and male sex and LDH level are associated with severe disease, and serum sodium is associated with mortality.

\section{Acknowledgements}

This research was conducted by the COVID-19 research group of CSAPG (led by Alejandro Rodríguez-Molinero; e-mail: rodriguez.molinero@gmail.com), which include, in addition to the authors of this paper, Alberti Casas, Anna PhD, MD; Borrego Ruiz, Manel BS; Campo Pisa, Pedro L.; Fenollosa Artés, Andreu MD; Hernandez Martinez, Lourdes MD; Hidalgo García, Antonio MD; Milà Ràfols, Núria RN; Molina Hinojosa, José C. MD; Monaco, Ernesto E. MD; Peramiquel Fonollosa, Laura MD; Pisani Zambrano, Italo G. MD; Rives, Juan P. MD; Sabria Bach, Enric MD; Sanchez Rodriguez, Yris M. MD; Segura Martin, Maria del Mar RN; Venturini Cabanellas, Florencia I. MD; Vidal Meler, Natalia MD; Macho Pérez, Oscar MD; López, Gabriela F. MD; Robles Portillo, María Teresa MD; Dapena Díaz, María Dolores MD; Martínez, Sergi MD, PhD; Rodríguez Gullello, Ezequiel A. MD; and Collado Pérez, Isabel MD. Group affiliations: Ârea de Recerca, Consorci Sanitari de l'Alt Penedès i Garraf, Vilafranca del Penedès, Barcelona (Spain).

We would like to thank Gloria Moes for her invaluable help in coordinating the fieldwork; Gloria Alba, Nuria Pola, and Anna María Soler for their initial help in collecting drug data; Montserrat Pérez and Rosa Guilera for their help with the electronic medical records; and David Blancas and Lourdes Gabarró for their work in the hospital protocols for COVID-19 and their initial supply of the bibliography. We also thank the CSAPG informatics team for their technical support during the study. Finally, we thank the manager of the Consorci Sanitari de l'Alt Penedès i Garraf, José Luis Ibáñez Pardos, and the management team, for making this study possible. 


\section{Statement of Ethics}

The present study has been performed in accordance with the Declaration of Helsinki, and it was approved by the Research Ethics Committee of the Hospital Universitari de Bellvitge (act 12/20, PR 252/20, date June 25, 2020), which approved the study without the need for the informed consent of the patients, given the observational nature of the study and the anonymous nature of the data collected.

\section{Conflict of Interest Statement}

The authors have no conflicts of interest to declare.

\section{Funding Sources}

This trial was developed without any external source of funding.

\section{Author Contributions}

César Gálvez-Barrón and Marta Arroyo-Huidobro analysed and interpreted the data and wrote the manuscript. Alejandro Rodríguez-Molinero designed the trial and analysed and interpreted the data. Antonio Miñarro analysed and interpreted the data. Gemma Añaños, Antonio, Chamero, Mireia Martín, Clara Gris, Jose L. Avalos, Anna M. Capielo, Ester Ventosa, and Gemma Tremosa collected and analysed the data. All authors read and approved the final manuscript. A preprint version of this article is available on Research Square [38].

\section{References}

1 Guo Y, Liu X, Deng M, Liu P, Li F, Xie N, et al. Epidemiology of COVID-19 in older persons, Wuhan, China. Age Ageing. 2020 Aug 24;49(5):706-12.

2 Bonanad C, García-Blas S, Tarazona-Santabalbina F, Sanchis J, Bertomeu-González V, Fácila $L$, et al. The effect of age on mortality in patients with COVID-19: a meta-analysis with 611,583 subjects. J Am Med Dir Assoc. $2020 \mathrm{Jul} ; 21(7): 915-18$.

3 High KP, Bradley SF, Gravenstein S, Mehr DR, Quagliarello VJ, Richards C, et al. Clinical practice guideline for the evaluation of fever and infection in older adult residents of long-term care facilities: 2008 update by the Infectious Diseases Society of America. J Am Geriatr Soc. 2009 Jan 15;48(2):149-71.

4 Samaras N, Chevalley T, Samaras D, Gold G. Older patients in the emergency department: a review. Ann Emerg Med. 2010 Sep;56(3): 261-9.

5 Norman DC, Yoshikawa TT. Fever in the elderly. Infect Dis Clin North Am. 1996 Mar; 10(1):93-9.

6 Lithander FE, Neumann S, Tenison E, Lloyd $\mathrm{K}$, Welsh TJ, Rodrigues JCL, et al. COVID-19 in older people: a rapid clinical review. Age Ageing. 2020 Jul;49(4):501-15.

7 Le Quintrec JL, Bussy C, Golmard JL, Hervé C, Baulon A, Piette F. Randomized controlled drug trials on very elderly subjects: descriptive and methodological analysis of trials published between 1990 and 2002 and comparison with trials on adults. J Gerontol A Biol Sci Med Sci. 2005;60(3):340-4.

8 Shenoy P, Harugeri A. Elderly patients' participation in clinical trials. Perspect Clin Res. 2015;6(4):184-9.
9 Ramos-Rincon J-M, Buonaiuto V, Ricci M, Martín-Carmona J, Paredes-Ruíz D, Calderón-Moreno $\mathrm{M}$, et al. Clinical characteristics and risk factors for mortality in very old patients hospitalized with COVID-19 in Spain. J Gerontol A Biol Sci Med Sci. 2021 Feb 25; 76(3):e28-37.

10 Covino M, De Matteis G, Santoro M, Sabia L, Simeoni B, Candelli M, et al. Clinical characteristics and prognostic factors in COVID-19 patients aged $\geq 80$ years. Geriatr Gerontol Int. 2020;20(7):704-8.

11 Gutiérrez Rodríguez J, Montero Muñoz J, Jiménez Muela F, Guirola García-Prendes C, Martínez Rivera M, Gómez Armas L. Variables associated with mortality in a selected sample of patients older than 80 years and with some degree of functional dependence hospitalized for COVID-19 in a Geriatrics Service. Rev Esp Geriatr Gerontol. 2020 NovDec;55(6):317-25.

12 Rodríguez-Molinero A, Gálvez-Barrón C, Miñarro A, Macho O, López GF, Robles MT, et al. Association between COVID-19 prognosis and disease presentation, comorbidities and chronic treatment of hospitalized patients. PLoS One. 2020 Oct 15;15(10): e0239571.

13 Berlin DA, Gulick RM, Martinez FJ. Severe Covid-19. N Engl J Med. 2020;383(25):245160.

14 WHO Working Group on the Clinical Characterisation and Management of COVID-19 infection. A minimal common outcome measure set for COVID-19 clinical research. Lancet Infect Dis. 2020;20:e192-7.

15 Benjamini Y, Hochberg Y. Controlling the false discovery rate: a practical and powerful approach to multiple testing. J Roy Statist Soc Ser B. 1995;57(1):289-300.
16 Tibshirani R. Regression shrinkage and selection via the Lasso. J Roy Statist Soc Ser B. 1996; 58(1):267-88.

17 Núñez E, Steyerberg EW, Núñez J. [Regression modeling strategies]. Rev Esp Cardiol. 2011;64(6):501-7.

18 Argenziano MG, Bruce SL, Slater CL, Tiao JR, Baldwin MR, Barr RG, et al. Characterization and clinical course of 1000 patients with coronavirus disease 2019 in New York: retrospective case series. BMJ. 2020 May 29;369:m1996.

19 Liang WH, Guan WJ, Li CC, Li YM, Liang HR, Zhao Y, et al. Clinical characteristics and outcomes of hospitalised patients with COVID-19 treated in Hubei (epicentre) and outside Hubei (non-epicentre): a nationwide analysis of China. Eur Respir J. 2020;55(6): 2000562.

20 Jiménez E, Fontán-Vela M, Valencia J, Fernandez-Jimenez I, Álvaro-Alonso EA, Izquierdo-García E, et al. Characteristics, complications and outcomes among 1549 patients hospitalised with COVID-19 in a secondary hospital in Madrid, Spain: a retrospective case series study. BMJ Open. 2020;10(11):e042398.

21 Annweiler C, Sacco G, Salles N, Aquino J-P, Gautier J, Berrut G, et al. National French survey of COVID-19 symptoms in people aged 70 and over. Clin Infect Dis. 2021 Feb 1;72(3): 490-4.

22 Zhao H-L, Huang Y-M, Huang Y. Mortality in older patients with COVID-19. J Am Geriatr Soc. 2020 Aug;68(8):1685-7.

23 Shahid Z, Kalayanamitra R, McClafferty B, Kepko D, Ramgobin D, Patel R, et al. COVID-19 and older adults: what we know. J Am Geriatr Soc. 2020;68(5):926-9. 
24 Mostaza JM, García-Iglesias F, GonzálezAlegre T, Blanco F, Varas M, HernándezBlanco C, et al. Clinical course and prognostic factors of COVID-19 infection in an elderly hospitalized population. Arch Gerontol Geriatr. 2020 Jul 27;91:104204.

25 Izcovich A, Ragusa MA, Tortosa F, Lavena Marzio MA, Agnoletti C, Bengolea A, et al. Prognostic factors for severity and mortality in patients infected with COVID-19: a systematic review. PLoS One. 2020;15(11): e0241955.

26 Malik P, Patel U, Mehta D, Patel N, Kelkar R, Akrmah M, et al. Biomarkers and outcomes of COVID-19 hospitalisations: systematic review and meta-analysis. BMJ Evid Based Med. 2020 Sep 15. Epub ahead of print.

27 Henry BM, de Oliveira MHS, Benoit S, Plebani M, Lippi G. Hematologic, biochemical and immune biomarker abnormalities associated with severe illness and mortality in coronavirus disease 2019 (COVID-19): a metaanalysis. Clin Chem Lab Med. 2020 Jun 25; 58(7):1021-8.

28 Zhang JJY, Lee KS, Ang LW, Leo YS, Young BE. Risk factors of severe disease and efficacy of treatment in patients infected with $\mathrm{CO}$ VID-19: a systematic review, meta-analysis and meta-regression analysis. Clin Infect Dis. 2020 Nov 19;71(16):2199-206.
29 Ou M, Zhu J, Ji P, Li H, Zhong Z, Li B, et al. Risk factors of severe cases with COVID-19: a meta-analysis. Epidemiol Infect. 2020 Aug 12; 148:e175.

30 Han Y, Zhang H, Mu S, Wei W, Jin C, Tong $\mathrm{C}$, et al. Lactate dehydrogenase, an independent risk factor of severe COVID-19 patients: a retrospective and observational study. Aging. 2020 Jun 24;12(12):11245-58.

31 Kuang ZS, Yang YL, Wei W, Wang JL, Long $\mathrm{XY}$, Li KY, et al. Clinical characteristics and prognosis of community-acquired pneumonia in autoimmune disease-induced immunocompromised host: a retrospective observational study. World J Emerg Med. 2020; 11(3):145-51.

32 Ding J, Karp JE, Emadi A. Elevated lactate dehydrogenase $(\mathrm{LDH})$ can be a marker of immune suppression in cancer: interplay between hematologic and solid neoplastic clones and their microenvironments. Cancer Biomark. 2017;19(4):353-63.

33 Gupta A, Madhavan MV, Sehgal K, Nair N, Mahajan S, Sehrawat TS, et al. Extrapulmonary manifestations of COVID-19. Nat Med. 2020;26(7):1017-32.
34 Berry SD, Ngo L, Samelson EJ, Kiel DP. Competing risk of death: an important consideration in studies of older adults. J Am Geriatr Soc. 2010;58(4):783-7.

35 Zerah L, Baudouin É, Pépin M, Mary M, Krypciak S, Bianco C, et al. Clinical characteristics and outcomes of 821 older patients with SARS-Cov-2 infection admitted to acute care geriatric wards. J Gerontol A Biol Sci Med Sci. 2021 Feb 25;76(3):e4-12.

36 Working group for the surveillance and control of COVID-19 in Spain. The first wave of the COVID-19 pandemic in Spain: characterisation of cases and risk factors for severe outcomes, as at 27 April 2020. Euro Surveill. 2020 Dec;25(50):2001431.

37 De Smet R, Mellaerts B, Vandewinckele H, Lybeert P, Frans E, Ombelet S, et al. Frailty and mortality in hospitalized older adults with COVID-19: retrospective observational study. J Am Med Dir Assoc. 2020 Jul;21(7): 928-32.e1.

38 Gálvez-Barrón C, Arroyo-Huidobro M, Miñarro A, Añaños G, Chamero A, Martín M, et al. COVID 19: clinical presentation and prognostic factors of severe disease and mortality in the oldest-old population. A cohort study. [Preprint]. 2021 Jan 21 [cited 2021 Jan 22]. Available from: https://www.researchsquare. com/article/rs-104338/v3 